\title{
EVALUACIÓN DE LOS ATRIBUTOS FISICOQUÍMICOS DE UVAS BRONZE "GRANNY VAL" MUSCADINAS DURANTE LA MADURACIÓN
}

\author{
AURORA JOSEFINA ESPINOZA ESTABA * \\ JUAN LUIS SILVA PACHECO ** \\ JESÚS RAFAEL MÉNDEZ NATERA *** \\ ATILANO LORENZO NÚÑEZ CALCAÑO****
}

\begin{abstract}
Se evaluó el efecto de la madurez sobre algunas características físicas, y composición química de uvas de la variedad 'Granny Val', utilizando frutos cosechados en tres diferentes estados de madurez: verde, de mesa y madura. Se estudiaron la variables: $\mathrm{pH}$, sólidos solubles (SS), acidez titulable (AT), SS/AT, azúcares (glucosa, sacarosa y fructosa), fenoles, color y firmeza. El análisis de las observaciones experimentales demostró que durante el proceso de maduración ocurrieron cambios que resultaron en el aumento cuantitativo de los valores de algunas variables: SS $(9,50$ a 12,80), la relación SS/AT $(2,06$ a 5,17) pH $(3,14$ a 3,44), compuestos fenólicos $(25,23$ a 31,96$)$, mientras que la AT disminuyó $(4,64$ a 2,48$)$. Los valores para el color de la superficie, Hunter $L^{*}$ disminuyeron, así como los de $b^{*}$ mientras que los de $a^{*}$ aumentaron. Las uvas, se oscurecieron y cambiaron de verde a amarillento. La firmeza (compresión), disminuyó en un 48 \% y la dureza de la concha en un $14 \%$ durante la maduración. Sin embargo, estas uvas conservan buenas características en su madurez que les permiten resistir los efectos del manejo y transporte.
\end{abstract}

PALABRAS CLAVE: UVAS; Vitis rotundifolia; MADURACIÓN; CALIDAD POSCOSECHA.

* Ph. D. Ciencia y Tecnología de los Alimentos, Mississippi State University, Estados Unidos, Profesor Titular, Ciencia y Tecnología de los Alimentos, Núcleo Monagas de la Universidad de Oriente, Maturín, Monagas, Venezuela (e-mail: aurorae51@hotmail.com).

** Ph. D. Ciencia y Tecnología de los Alimentos, Mississippi State University, Estados Unidos, Profesor, Food Processing and Safety, Mississippi State University, Starkville, Mississippi, Estados Unidos (e-mail: jls46@msstate.edu).

*** Doctorando en Ciencias Agrícolas, Universidad Central de Venezuela, M. Sc. Agricultura Tropical, Profesor Titular, Departamento de Agronomía, Núcleo Monagas, Universidad de Oriente, Maturín, Monagas, Venezuela (e -mail: jmendezn@cantv.net).

**** Ph. D. Ciencia y Tecnología de los Alimentos, Mississippi State University, Estados Unidos, Profesor Titular, Control de la Calidad, Núcleo Monagas, Universidad de Oriente, Maturín, Monagas, Venezuela (e-mail: alnunezc@gmail.com). 


\section{INTRODUCCION}

El género Vitis contiene dos subgéneros, Euvitis Planch (uvas de racimo) y Muscadinia Planch (uvas muscadinas). La uvas muscadina (Vitis rotundifolia Michx.) es el único miembro comúnmente cultivado del subgénero Muscadinia (CONNER, 2009).

La evaluación de las variables físico-químicas que influyen en la madurez de la uva son factores importantes para la calidad de la uva de mesa y del vino, ya que la cosecha acertada determina el potencial de su calidad. Las evaluaciones de la madurez no son una tarea fácil; los cultivadores deben entender que la calidad de vino es en gran parte dependiente no sólo de compuestos primarios, sino también de metabolitos secundarios que afectan el aroma (ZOECKLEIN, 2001). El consumo de la uva incluye variedad de formas desde fresca hasta procesadas. El grado de madurez necesario varía dependiendo del uso del procesador y del consumidor. Es por eso que el proceso de maduración de uvas es importante y éste por ser un fruto no climatérico, ocurre mientras el fruto permanece en la planta. A pesar de su importancia económica existen pocas investigaciones e inversiones para determinar y entender los mecanismos moleculares relacionados con los efectos de la maduración en la calidad de uvas. La calidad puede variar de año en año y ésta inconsistencia puede ser atribuida al suelo, a cambios en el microclima y a las prácticas en el manejo del cultivo. Los aspectos genéticos que determinan la calidad de las uvas son prácticamente desconocidos, aún más cómo interactúan los factores externos a nivel de los genes para causar diferencias en la calidad está por conocerse.

El crecimiento, desarrollo, maduración, senescencia y muerte celular en las frutas es un proceso complejo que va acompañado de diversas transformaciones y cambios bioquímicos y metabólicos. Estos cambios ocurren de manera continua y provocan cambios en los componentes químicos, en las características fisicas y sensoriales (DOREYAPPA y HUDDAR, 2001) dando lugar a un fruto más atractivo pero también conducen al deterioro y muerte. Estudiar las características físico-químicas y los atributos de los frutos permite conocer mejor su comportamiento para efectos de su cosecha, almacenamiento, vida útil y procesamiento, por lo que la determinación de variables de calidad se han convertido en estudios esenciales en la medida en que se realizan más investigaciones en el área de genética, mejoramiento, y mecanismos bioquímicos de la maduración y ablandamiento. A todo esto se debe agregar el marcado interés en la actualidad para el establecimiento o fijación de índices de maduración, así como el potencial de almacenamiento de frutos bajo condiciones variadas (LAGUADO et al., 1998) y el hecho de que las frutas son cosechadas y almacenadas en ocasiones causando stress fisiológico que conduce a cambios y efectos adversos en su apariencia y perfil químico (KALT, 2005). Existen muy pocos estudios físico-químicos en poscosecha relacionados con cambios durante la maduración de uvas. La mayoría de las investigaciones están relacionadas con los tipos Vitis vinifera y $V$. labrusca, y poca información relacionada con el tipo $V$. rotundifolia objeto de esta investigación. Una de las variedades de uva verde es 'Granny Val' y se caracteriza por su alto rendimiento y su alta resistencia a manejo, haciéndola una variedad de excelente uso comercial (BRADY et al., 2010). El control del tiempo de cosecha, tamaño de la fruta y color, el nivel de acidez y la cantidad de compuestos volátiles y aroma en uvas de mesa y para vino son una de las mayores preocupaciones de los cultivadores. La mejor calidad del mosto se obtiene cuando la cosecha se realiza en el momento de la madurez apropiada según el cultivar. En el trópico el proceso de maduración es muy desigual (OJEDA y PIRE, 1997). En uvas, no se conoce con exactitud el mecanismo que desencadena la segunda fase de su maduración.

El objetivo de este trabajo fue determinar la calidad física y química de las uvas muscadinas de la variedad 'Granny Val' cosechadas a diferentes estados de maduración.

\section{MATERIAL Y MÉTODOS}

Se utilizaron uvas muscadinas de la variedad 'Granny Val' cultivadas en la Estación Experimental de Cristal Spring, Mississippi. Las uvas fueron recogidas a mano en tres estados 
diferentes de maduración, verde, de mesa y madura (para jugo o vino).

Las frutas se dividieron en cinco replicaciones para cada estado de madurez. Se utilizaron $200 \mathrm{~g}$ de muestra para las mediciones de los sólidos solubles SS ('Brix), $\mathrm{pH}$, acidez titulable (AT), relación SS/AT, azúcares individuales (sacarosa, glucosa, fructosa), fenoles totales, color de la superficie $L^{*} a^{*} b^{*}$, firmeza (fuerza de compresión, $F C$ ), y dureza de la concha (fuerza de penetración, $\mathrm{FP})$.

\subsection{ANÁLISIS QUÍMICO}

Las muestras de jugo utilizadas para la medición de las variables, SS ('Brix), azúcares, $\mathrm{pH}$, acidez titulable (AT), relación SS/AT, fenoles se preparó triturando $200 \mathrm{~g}$ de uvas en un mortero, el jugo fue filtrado utilizando tela de liencillo en tres capas.

Sólidos solubles (SS) ( $\left.{ }^{\circ} \mathrm{Brix}\right)$ : Se determinaron por refractometría con refractómetro modelo ABBE 3L Refractometer mantenido a $22^{\circ} \mathrm{C}$ (BAUSCH \& LOMB, 2000).

pH del jugo: Se determinó mediante un pHmetro Accumet pH 925 (FISHER SCIENTIFIC, 1987).

Acidez titulable (AT): Se expresa como porcentaje de ácido tartárico/100 mL jugo. Se determinó por medición directa y potenciométrica. A $5 \mathrm{~mL}$ de jugo se agregaron 3 gotas de fenolftaleína y se utilizó $\mathrm{NaOH}$ 0,1 N para titular, hasta el punto final de 8,2 (WOODS y AURAND, 1977):

$$
\mathrm{AT}=\frac{(\mathrm{mL} \mathrm{NaOH})(\mathrm{N} \mathrm{NaOH})(\text { meq ácido) }}{\text { Volumen de la muestra }} \times 100 \quad \text { (meq ácido tartárico: 0,075) }
$$

Relación SS/AT: Los valores de SS divididos por los valores obtenidos para AT.

Fenoles Totales. Se clarificó el jugo por $12 \mathrm{~h}$ a una temperatura de $4{ }^{\circ} \mathrm{C}$. Para cuantificar los fenoles, se utilizó el método de Singleton y Rossi (1965). Las muestras se colocaron en reposo para hasta desarrollar un color azul (< de una hora). Se midió la absorción a una longitud de onda de $765 \mathrm{~nm}$ en un Espectrofotómetro Perkin Elmer UV/VIS Lambda 3B, previamente calibrado. EI contenido de fenoles se calculó mediante la curva estándar calculada con ácido gálico y los datos se expresan como mg de ácido gálico/100 mL (Sigma, St. Louis, MO).

\subsection{ANÁLISIS FÍSICO}

El color de la superficie de las uvas se determinó mediante un Colorímetro Modelo Hunter Labscan 6000 0/45 (Hunter Associates Laboratory, Reston VA). El valor de "L" mide el grado de luminosidad, el valor de "a" mide el grado de rojo cuando es positivo $(a+) y$ verde cuando es negativo $(a-)$ y los valores de ' $b$ ' miden el grado de amarillo cuando es positivo $(b+)$ y grado de azul cuando es negativo (b-) (POMERANS y MELOAN, 1978). Los valores se expresan como promedio de cinco mediciones en diferentes puntos de la fruta.

Prueba de compresión: Se utilizó el equipo Instron, Modelo 1011 Instron (Universal Testing Machine, Canton, MA) con una punta cilíndrica de $10 \mathrm{~mm}$ y una velocidad de $50 \mathrm{~mm} / \mathrm{min}$ y una escala de rango de $5 \mathrm{~N}$ hasta la penetración de cada fruta (se realizó la medición de 10 frutas y se reporta el promedio). La fuerza máxima se computó solo cuando las uvas empezaron a liberar jugo (INSTRON, 1972).

Prueba de penetración: Se utilizó el equipo Instron Modelo 1011 (Instron, Universal Testing Machine, Canton, MA) con una punta de $2 \mathrm{~mm}$ y una velocidad de $50 \mathrm{~mm} / \mathrm{min}$ con un rango de carga de $5 \mathrm{~N}$. La punta se desplazó hasta la penetración de cada fruta (se utilizó el promedio de 10 frutas) (INSTRON, 1972). 


\subsection{ANÁLISIS ESTADÍSTICO}

Se utilizó un diseño completamente aleatorizado, con cinco repeticiones, se aplicó PROC ANOVA y para las diferencias significativas $(p \leq 0,05)$ se utilizó la prueba de la Mínima Diferencia Significativa (MDS) (SAS, 1997; STEEL y TORRIE, 1985). Se realizó un análisis de correlación lineal simple ( $r$ ) de Pearson entre pares de caracteres, a un nivel de probabilidad de $p \leq 0,01$ y $p \leq 0,05$. Se utilizó el paquete estadístico SPSS versión 12.0 (SPSS, 2003).

\section{RESULTADOS Y DISCUSIÓN}

\subsection{CARACTERÍSTICAS QUIIMICAS}

Los resultados obtenidos para la variable $\mathrm{pH}$ se muestran en el Cuadro 1. Se observó un aumento significativo en el pH durante la madurez (10\%). Huang (1996), reportó valores de 3,07 para uvas 'Granny Val' en el estado de mesa. Resultados similares fueron reportados por Watson (2003) quien señaló valores de pH de 3,18, 3,34 y 3,48 para uvas maduras a diferentes tiempos de cosecha y Walker et al. (2001) reportaron un incremento del pH 3,33 a 3,46 \% en uvas muscadinas de la variedad Fry a medida que los frutos maduraban. Los cambios de $\mathrm{pH}$ en las uvas durante la madurez han sido reportados en diferentes investigaciones y son causados por el metabolismo de los ácidos y la acumulación de cationes (HRAZDINA, PARSONS y MATTICK, 1984). Breman et al. (2007) en un estudio con 11 cultivares de uvas muscadinas en el Norte de Florida, Estados Unidos, reportaron un rango de $\mathrm{pH}$ entre 3,00 y 3,56 con un valor de 3,09 para la variedad 'Granny Val' (penúltimo menor valor), siendo similar al pH obtenido para el grado de madurez verde en este ensayo. Estos autores indicaron que mientras menor el $\mathrm{pH}$, mayor fue la preferencia del consumidor para el cultivar. Stringer et al. (2008) en un ensayo con 35 cultivares de uvas muscadinas en McNeil, Mississippi, Estados Unidos durante 2001, 2002 y 2006, encontraron un rango de pH entre 3,2 (cultivar 'Scuppernong') y 4,1 (cultivar 'Dixie'), los valores de pH obtenidos en este estudio para los tres estados de maduración se encuentra dentro de este rango. Bubola y Peršurić (2012) indicaron valores de $\mathrm{pH}$ de 3,39 y 3,55 para los años 2009 y 2010 , respectivamente en el cultivar 'Istrian Malvasia de Vitis vinífera, mientras que Chiarotti et al. (2011) reportaron un valor de 3,53 y 3,55 para las zafras 2009/2010 y 2010/2011, respectivamente en el cultivar cv. Bordô y Jogaiah et al. (2013) indicaron un $\mathrm{pH}$ de 3,27 para uvas Sauvignon Blanc y 1,07 para uvas Cabernet Sauvignon como promedio de dos años. En esta investigación, el mayor pH se encontró en las uvas maduras $(3,44)$, lo cual es una buena característica porque de acuerdo a Kodur (2011), el pH del jugo de uvas > a 3,8 es indeseable para la producción de vinos de calidad porque producen vinos de baja calidad, por ejemplo, una estabilidad reducida del color y un sabor pobre.

\section{CUADRO 1 - VALORES PROMEDIOS PARA LAS VARIABLES FÍSICO-QUÍMICAS DE UVAS 'GRANNY VAL' EN TRES ESTADOS DE MADUREZ}

\begin{tabular}{|lccccc|}
\hline Madurez & pH & $\begin{array}{c}\text { Sólidos } \\
\text { Solubles } \\
\text { SS (\%) }\end{array}$ & $\begin{array}{c}\text { Acidez Titulable } \\
\text { (\% ácido tartárico) }\end{array}$ & SSIAT & $\begin{array}{c}\text { Fenoles } \\
\text { (mg ácido } \\
\text { gálico/100 mL) }\end{array}$ \\
\hline Verde & $3,14 \mathrm{c}$ & $9,55 \mathrm{c}$ & $0,46 \mathrm{a}$ & $20,7 \mathrm{c}$ & $25,24 \mathrm{c}$ \\
Mesa & $3,28 \mathrm{~b}$ & $10,94 \mathrm{~b}$ & $0,33 \mathrm{~b}$ & $33,6 \mathrm{~b}$ & $28,88 \mathrm{~b}$ \\
Madura & $3,44 \mathrm{a}$ & $12,82 \mathrm{a}$ & $0,25 \mathrm{c}$ & $51,8 \mathrm{a}$ & $31,97 \mathrm{a}$ \\
\hline MDS $(0,05)$ & 0,09 & 0,82 & 0,03 & 3,2 & 2,36 \\
\hline
\end{tabular}

a, b, c. Medias con letras diferentes dentro de las columnas difieren $(p \leq 0,05)$ de acuerdo a la prueba de la Mínima Diferencia Significativa (MDS). 
Los sólidos solubles (SS) aumentaron de 9,55 a 12,82 (30 \%) durante la maduración, estos valores están por debajo del rango indicado por Conner (2009) de 12,4 y $16,8 \%$ en 23 cultivares de muscadinas, a excepción del estadio maduro y por debajo de los reportados por Stringer et al. (2008) quienes en un ensayo con 35 cultivares de uvas muscadinas encontraron un rango de SS entre $13,5 \%$ (cultivar 'Regale') y 18,6 \% (cultivar 'Dixie'). Estas diferencias pueden haber resultado de una combinación de factores ambientales que afectan la calidad de la baya y la prontitud de las cosechas las cuales fueron necesarias para minimizar el daño de las abejas y avispas a los frutos (STRINGER et al., 2008), así como también a la utilización de diferentes genotipos. Los SS se incrementaron a medida que los frutos maduraban, similares resultados encontraron Lanier and Morris (1979) en las uvas muscadinas de la variedad Carlos de 8,0 a $15 \%$ en cinco estadios de maduración. Walker et al. (2001) reportaron un incremento de los SS de 14,2 a 19,5\% en uvas muscadinas de la variedad Fry del estadio 1 al 5.

Morris y Brady (2004) indicaron que el porcentaje de sólidos solubles y de ácido tartárico son dos parámetros comúnmente utilizados para determinar la madurez de la uva. El contenido de SS en uvas es una buena medida o índice para determinar la madurez y es también la base para los cálculos de la producción potencial de alcohol y la necesidad de amelioración durante la vinificación. Aproximadamente $90-95 \%$ de los SS en las uvas maduras son azúcares fermentables, principalmente glucosa y fructosa. Normalmente durante la maduración de las frutas aumentan los SS, este hecho puede ser atribuido al rompimiento de las moléculas de almidón, a su transformación a azúcares solubles y a la disminución de los ácidos. Huang (1996) reportó valores de 9,52 Brix para uvas 'Granny Val' de mesa y Chamul (1990) señaló valores de 12,9 'Brix para jugos de uvas bronze muscadinas maduras, tal como en este estudio. Por el contrario, Andersen (2006) evaluó 20 cultivares de uvas muscadinas durante cuatro años (2002-2005) e indicó un valor promedio de $17,0^{\circ}$ Brix en el jugo de los frutos de 'Granny Val', siendo uno de los cultivares que produjo frutos con los más altos sólidos solubles. Considerando los valores de ${ }^{\circ}$ Brix igual o mayor a 11 como dulces (ASENJO, 1980), en este caso los frutos de mesa y maduros presentaron esta característica, los verdes presentaron valores menores de $11^{\circ}$ Brix. Estos resultados coinciden con los de Flora y Lane (1979); Morris (1980) y Philip y Kuyenda (1973). Breman et al. (2007) indicaron un rango de sólidos solubles ('Brix) entre 12,60 y 21,45 con un valor de 13,50 para la variedad 'Granny Val' (antepenúltimo menor valor), siendo mayor a todos los valores de SS obtenidos para los diferentes grados de madurez en este ensayo. Basha et al. (2012) reportaron un valor de $10 \%$ SS para Granny Val, menor que el reportado en este trabajo.

Los valores obtenidos para la variable AT disminuyeron durante la maduración, el estado verde de 0,46 hasta 0,25 en el estado maduro esto representa un 46,5\%. Mientras los azúcares aumentaron la acidez disminuyó. Morris y Brady (2004) indicaron un incremento de los azúcares y la disminución de los ácidos durante la maduración normal de las uvas. Watson (2003) reportó variaciones de valores de ácido tartárico y málico de 3-7 g/L (0,3-0,7 \%) y 1-5 g/L (0,1-0,5\%), respectivamente y variaciones de acuerdo con la madurez, variedad, cultivar y clima. Huang (1996) señaló que la AT disminuyó del estado verde al maduro, resultados iguales al obtenido en esta investigación, así como los indicados por Lanier and Morris (1979) en las uvas muscadinas de la variedad Carlos, los cuales variaron de 1,87 a $0,92 \%$ de ácido tartárico en cinco estadios de maduración y Walker et al. (2001) señalaron una disminución de AT de 0,65 a 0,58 \% de ácido tartárico en uvas muscadinas de la variedad Fry del estadio 1 al 5. Carrol, Hoover y Nesbitt (1971) indicaron valores bajos de AT para uvas 'Topsail', mientras Breman et al. (2007) reportaron un rango de 0,16 a $0,59 \%$ de ácido tartárico en 11 cultivares de uvas muscadinas al Norte de Florida con un valor de 0,29\% para 'Granny Val', valor dentro del rango obtenido en este ensayo. Los ácidos predominantes en uvas son: el málico y el tartárico y menor proporción de cítrico y oxálico. El ácido málico es considerado un ácido frágil, que puede ser fácilmente degradado por microorganismos presentes en los frutos; éste se encuentra en la uva verde y tiende a disminuir en la madurez. El cítrico es un ácido poco abundante en la uva y al igual que el málico es fácilmente degradado por 
las bacterias lácticas y tiende a desaparecer. El tartárico por el contrario es un ácido más fuerte, más específico en la uva y el vino y representa la tercera o cuarta parte de los ácidos del vino (KENNEDY, 2002). El ácido tartárico y el málico representan cerca del $90 \%$ de los ácidos de las uvas para vino. Ambos ácidos son productos secundarios del metabolismo de los azúcares y aumentan rapidamente a medida que la fruta madura. Después de la cosecha el contenido de ácido tartárico disminuye debido al aumento del tamaño del fruto y permanece relativamente constante durante los últimos estadios de la madurez. El ácido málico disminuye también como consecuencia del aumento del tamaño de la fruta pero además debido a la utilización como fuente de energía durante la respiración. Estas consideraciones explican en parte la disminución de AT por la disminución del ácido málico y el cítrico y presencia del tartárico durante la maduración. Philip y Kuyenda (1973), reportaron que el $\mathrm{pH}$ y ${ }^{\circ}$ Brix aumentaron de manera estable con una disminución en AT durante el desarrollo de las uvas 'Thompson Seedless'. Los SS, el nivel de acidez y el pH son variables utilizadas como indicadores de madurez para uvas en la fabricación de vino. La mayor aceptación del consumidor generalmente se logra con frutas de alto contenido de SS. La AT y el pH son factores importantes en la cosecha debido a que afectan la acidez y la estabilidad microbiológica y química de los vinos. Morris y Brady (2004) señalaron que la evaluación del color de la baya y los contenidos de azúcar y ácido mostraron que la madurez de la fruta se incrementó con la densidad en aumento, a medida que la fruta madura, hay un incremento correspondiente en el porcentaje de sólidos solubles y una disminución de la acidez titulable, expresada en ácido tartárico

La relación SS/AT conocida por algunos autores como índice de madurez se muestra con un rango entre 20,6 para las uvas verdes a 51,7 en las uvas maduras, un aumento del $150 \%$ durante la madurez. Breman et al. (2007) en un estudio con 11 cultivares de uvas muscadinas señalaron un rango de la relación SS/AT entre 21,33 y 100,31 con un valor de 46,55 para la variedad 'Granny Val' (antepenúltimo menor valor), estando este valor dentro del rango de esta investigación, acercándose al valor de la fruta madura. Mascarenhas et al. (2010) evaluaron las características de uvas de mesa Benitaka, Festival, Isabel e Itália y hallaron una relación SS/AT de 18,50; 34,93; 17,12 y 19,87, respectivamente, valores inferiores a los encontrados en esta investigación para el estado maduro. Por otra parte, Mascarenhas et al. (2012) indicaron que las uvas BRS Clara y BRS Linda con una relación SS/AT cercana a 35,0 unidades tuvieron la mayor calidad seguida de BRS Morena, la cual mostró una buena relación SS/AT $(30,4)$, mientras que las uvas Advanced Selection 8 y Crimson presentaron las relaciones más bajas con 23,99 y 23,35, respectivamente. Por lo que las uvas maduras encontradas en esta investigación son de superior calidad con una relación SS/AT de 51,8 unidades, inclusive en la uvas de mesa mostraron una buena relación con 33,6. Simonne et al. (2005) encontraron que mientras mayor fue la relación SS/AT, los tomates (Lycopersicon esculentum) fueron más dulces y tendieron a recibir una mayor puntuación de preferencia por los consumidores, mientras que Breman et al. (2007) reportaron resultados diferentes, es decir, la relación SS/AT mostró un orden similar al de la preferencia del consumidor.

Los fenoles aumentaron a medida que las frutas maduraron en un $27 \%(25,24$ a 31,97 $\mathrm{mg}$ ácido gálico/100 mL). Striegler et al. (2005) reportaron un valor de $304 \mathrm{mg} / \mathrm{L}(30,4 \mathrm{mg} / 100 \mathrm{~mL})$ de fenoles totales en la variedad 'Granny Val', muy similar al obtenido en esta investigación para las uvas maduras. Mahdavi et al. (2010) indicaron valores de fenoles totales (mg de ácido gálico por $100 \mathrm{~mL}$ ) de 144,56 y 135,2 en jugos frescos naturales y jugos comerciales, respectivamente para uvas roja, 37,69 y 33,71 , respectivamente para uvas blancas y 49,40 y 41,81 , respectivamente para frutos de uvas rojas, valores superiores a los obtenidos en esta investigación. El análisis los fenoles es un requerimiento importante en la evaluación de las uvas y los vinos. Estos aumentan con el avance de la madurez, especialmente en la concha. Estos factores son importantes desde el punto de vista cuantitativo, pero es el aspecto cualitativo el que mas efecto tiene cuando se refiere a fenoles (ZOECKLEIN, 2001), ya que son la fuente principal del aroma, sabor, color y gusto de la uva, cualidades que contribuyen a la calidad del vino y deben ser considerados de manera especial en la evaluación de la madurez. Por otro lado, se considera que el contenido de fenoles emerge 
como un componente clave como factor de definición de la madurez de las uvas y otras frutas (GONZÁLEZ-SAN JOSÉ et al., 1991; CHAOVANALIKIT y WOROLSTAD, 2004 a,b). Chamul (1990), reportó valores de $31 \mathrm{mg}$ ácido gálico/100 mL de jugo de uvas muscadinas maduras, lo cual coincide con los valores en esta investigación. Diferentes autores han reportado valores similares entre 16,73 a 31,80 para vinos blancos (SIMONETTI, PIETA y TETOLIN, 1997).

En vinos, los principales compuestos fenólicos son el ácido cafeíco, ácidos cinámicos, flavonoides, epicatequina, catequina, ácido gálico, cianidina, procianidina, malvidina-glucósido, rutina, mirecitina, quercitina, resveratrol (KINSELLA et al., 1993). Estos fenoles además de contribuir a las características organolépticas del vino poseen propiedades antioxidantes. Varias investigaciones se avocan en la actualidad a reportes en el rango en que los fenoles son considerados importantes antioxidantes especialmente en uvas. Generalmente la composición fenólica depende de muchos factores, la variedad, proceso de elaboración, extracción y tiempo de almacenamiento y los valores en el vino son menores que para el jugo de uva fresca. Algunos fenoles también son compuestos antimicrobianos y aumentan a medida que los mecanismos de defensa de la uva lo hacen. En esta investigación el jugo de uva se extrajo de la maceración de las uvas con su concha, los valores presentados son el resultado en ambos tejidos. Es posible que el contenido de fenoles en uvas muscadinas no sea tan alto como ocurriría para uvas de la variedad vinífera, probablemente debido a su color bronceado y la variedad vinífera presenta color morado; sin embargo fenoles poco coloreados también contribuyen sustancialmente como antioxidantes y aún están en frutas verdes (KALT, 2005). Se cita en algunas investigaciones el aumento de concentración de (fitoalexina) resveratrol (SIEMANN y CREASY, 1992), uno de los polifenoles del vino que más atención ha suscitado en los últimos años. En uvas, la síntesis de resveratrol y otros stilbenos es estimulado cuando las frutas son infestadas por hongos (ROMERO-PÉREZ et al., 2001). Debido a que este compuesto actúa como fungicida y es inducido y estimulado por las infecciones en las uvas y sus niveles son muy variables (JEANDET et al., 1995; FRANKEL, WATERHOUSE y TEISSEDRE, 1995; INFANTE, 1997).

\subsection{CARACTERÍSTICAS FÍSICAS}

Los valores para Hunter 'L' (luminosidad) (Cuadro 2) disminuyeron de 41,58 a 36,55 a medida que las uvas maduraron, indicando un mayor oscurecimiento (12,09 \%). Flora y Lane (1979) reportaron cambios progresivos de oscurecimiento en la concha de uvas 'Cowart' y establecieron que el color de la concha y la relación SS/AT podría ser usada como indicador de madurez óptima. Los valores de Hunter 'a' (Cuadro 2) aumentaron y los de Hunter 'b' presentaron tendencia a disminuir. Liang, Chen y Hegwood (1991) encontraron que todos los cultivares aumentaron en grado de enrojecimiento pero disminuyeron en su amarillez durante el período de investigación. Similares resultados encontraron Lanier and Morris (1979) en las uvas muscadinas de la variedad Carlos donde el color se incrementaba a medida que la fruta maduraba.

CUADRO 2 - VALORES PROMEDIOS DE LAS VARIABLES FÍSICAS DE UVAS 'GRANNY VAL' EN TRES ESTADOS DE MADUREZ

\begin{tabular}{|lccccc|}
\hline \multirow{2}{*}{ Madurez } & $\begin{array}{c}\text { Fuerza de } \\
\text { Penetración (N) }\end{array}$ & $\begin{array}{c}\text { Fuerza de } \\
\text { Compresión (N) }\end{array}$ & \multicolumn{3}{c|}{ Color "Hunter" } \\
\cline { 4 - 6 } & $20,52 \mathrm{a}$ & $37,30 \mathrm{a}$ & $41,58 \mathrm{a}$ & $-3,05 \mathrm{~b}$ & $14,73 \mathrm{a}$ \\
Verde & $19,82 \mathrm{a}$ & $28,40 \mathrm{~b}$ & $39,46 \mathrm{~b}$ & $-1,83 \mathrm{~b}$ & $13,57 \mathrm{~b}$ \\
Mesa & $17,24 \mathrm{~b}$ & $19,28 \mathrm{c}$ & $36,56 \mathrm{c}$ & $1,66 \mathrm{a}$ & $13,39 \mathrm{~b}$ \\
\hline Madura & 2,20 & 6,23 & 0,92 & 1,58 & 0,903 \\
\hline
\end{tabular}

a, b, c. Medias con letras diferentes dentro de las columnas difieren $(p \leq 0,05)$ de acuerdo a la prueba de la Mínima Diferencia Significativa (MDS). 
La industria de la uva de mesa está consciente de la importancia de la firmeza de las bayas (CALLEJAS RODRÍGUEZ et al., 2011). La FC mide la dureza, mientras que la FP determina la firmeza de las bayas. La FC (Cuadro 2) disminuyó $(p \leq 0,05)$ en $47 \%$ mientras la frutas maduraron. El ablandamiento de bayas durante la maduración de la fruta se debe, específicamente, a la solubilización de polisacáridos pécticos de la pared celular y por la pérdida de residuos de galactosa desde las cadenas laterales de pectina (REDGWELL et al., 1997). Resultados similares fueron señalados por Walker et al. (2001) quienes reportaron una disminución de la firmeza de 10,01 a $7,97 \mathrm{~N}$ en uvas muscadinas de la variedad Fry a medida que los frutos maduraban. Por otro lado, la hidrólisis de carbohidratos políméricos, especialmente sustancias pécticas y hemicelulosa conlleva al debilitamiento de las paredes celulares y las fuerzas cohesivas que mantienen a las células unidas, esto se traduce en aumento de SS y pérdida de firmeza, la fuerza requerida para producir la ruptura en la compresión de las frutas, la cual disminuye cuando aumenta la maduración como sugieren varios autores (LIANG, CHEN y HEGWOOD, 1991; FILS-LYCAON y BURET, 1990). Stringer et al. (2008) señalaron que una mayor firmeza de las bayas es también importante en la reducción de la incidencia de daños de las frutas al momento de la cosecha y durante el transporte y finalmente resulta en una mayor calidad de almacenamiento y en un ensayo con 35 cultivares de uvas muscadinas encontraron una correlación significativa entre la firmeza de las bayas y la resistencia a las enfermedades de pudrición de las bayas.

Los resultados de la FP de las uvas verdes fue mayor $(20,52 \mathrm{~N})$ que para las maduras $(17,24 \mathrm{~N})$, es decir, la FP disminuyó con la maduración de las bayas. Estas uvas tienen una concha dura, por lo que deben presentar menores problemas cuando son cosechadas y manipuladas, así también debe mantener mejor su calidad y buen potencial para mantenerse sana durante el transporte.

\subsection{CORRELACIÓN LINEAL ENTRE LAS VARIABLES FÍSICO-QUÍMICAS}

La correlación entre las variables estudiadas se muestra en el Cuadro 3. Se observa que el $\mathrm{pH}$ presentó correlación negativa significativa con $A T, F C, L^{*}$ y b* y positiva significativa con $S S, a^{*}$ y fenoles. La AT disminuyó al aumentar el pH lo cual implica que se redujeron los ácidos y aumentaron los azúcares, similares resultados reportó Huang (1996) indicando valores de AT inversos a los de SS ('Brix) y pH para uvas 'Granny Val'.

CUADRO 3 - COEFICIENTES DE CORRELACIÓN ENTRE LAS VARIABLES FÍSICO-QUÍMICAS

\begin{tabular}{|c|c|c|c|c|c|c|c|c|}
\hline Variables & $\mathrm{pH}$ & AT & SS & FC & FP & $L^{*}$ & $a^{*}$ & $\mathbf{b}^{*}$ \\
\hline \multicolumn{9}{|l|}{$\mathrm{pH}$} \\
\hline AT & $-.838\left(^{* *}\right)$ & & & & & & & \\
\hline SS & $.780\left(^{* *}\right)$ & $-.877\left({ }^{* *}\right)$ & & & & & & \\
\hline $\mathrm{FC}$ & $-.671\left(^{* *}\right)$ & $.906\left(^{* \star}\right)$ & $-.863\left({ }^{* *}\right)$ & & & & & \\
\hline $\mathrm{FP}$ & -.398 & .417 & $-.608\left(^{*}\right)$ & $.657\left({ }^{* *}\right)$ & & & & \\
\hline$L^{*}$ & $-.889\left(^{* *}\right)$ & $.908\left(^{* *}\right)$ & $-.882\left({ }^{* *}\right)$ & $.799\left(^{* *}\right)$ & .461 & & & \\
\hline$a^{*}$ & $.830\left(^{* *}\right)$ & $\left.-.791{ }^{(* *}\right)$ & $.924\left({ }^{* *}\right)$ & $-.754\left({ }^{* *}\right)$ & $-.636\left(^{*}\right)$ & $-.896\left(^{* *}\right)$ & & \\
\hline$b^{*}$ & $-.570\left(^{*}\right)$ & $.671\left(^{* *}\right)$ & -.422 & $.531\left(^{*}\right)$ & .179 & $.591\left(^{*}\right)$ & -.346 & \\
\hline Fenoles & $.840\left(^{* *}\right)$ & $-.826\left(^{* *}\right)$ & $.878\left({ }^{* *}\right)$ & $-.797\left(^{* *}\right)$ & $-.623\left(^{*}\right)$ & $-.823\left(^{* *}\right)$ & $.924\left({ }^{* *}\right)$ & -.435 \\
\hline
\end{tabular}

* Correlación significativa $(p \leq 0,05) ; *$ * Correlación altamente significatia $(p \leq 0,01)$.

AT = Acidez titulable; SS = Sólidos solubles; FC = Fuerza de compresión; FP = Fuerza de penetración; Hunter Color L*, $a^{*}$, $b^{*}$. 
La concha de las uvas fue más blanda y la fuerza $(\mathrm{N})$ fue menor sin importar el estado de maduración. La disminución de los ácidos presentes en el fruto ayudan a la disminución de las fuerzas de sostén y de la cohesión entre los espacios intercelulares de las células en el fruto maduro, así mismo reducción de los puentes de calcio por lo que FC y FP disminuye. El color de la superficie $L^{*}$ presentó una correlación negativa con el pH. Lo mismo ocurrió con el valor de b*. La correlación del $\mathrm{pH}$ fue positiva con $\mathrm{a}^{*} \mathrm{y}$ los fenoles indicando un mayor grado de rojo, menos verde y mayor contenido de fenoles. La mayoría de las investigaciones realizadas en frutas y vegetales indican que al aumentar los SS, la acidez disminuye. Resultados similares para algunas asociaciones fueron reportados por Mascarenhas et al. (2012) quienes estudiaron la correlación entre las características de calidad de cinco cultivares de uva de mesa y encontraron una asociación significativa y negativa entre el pH con los sólidos solubles, acidez titulable, relación SS/AT y firmeza, mientras que los SS se asociaron positivamente con la relación SS/AT y firmeza. La AT se asoció negativamente con la relación SS/AT y positivamente con la firmeza.

\section{CONCLUSIÓN}

El proceso de maduración envolvió numerosas actividades en las uvas resultando en cambios en las variables estudiadas. En el estado maduro las uvas presentaron valores mayores de $\mathrm{pH}, \mathrm{SS}$, SS/AT, fenoles y menores para AT que en el estado verde, indicando cambios a mayor dulzura y menor acidez. Se observó una disminución en firmeza durante la maduración. El color de la superficie de las uvas cambió a amarillento bronceado y se oscurecieron cuando maduraron. La maduración fue gradual, los cambios en las variables no fueron bruscos como era de esperarse en un fruto no climatérico. Las uvas aún fueron más dulces mientras maduraron pero aún maduras indican una uva firme y resistente para los efectos de almacenamiento y transporte.

\section{ABSTRACT \\ PHYSICAL AND CHEMICAL ATTRIBUTES OF BRONZE MUSCADINES “GRANNY VAL" GRAPES DURING MATURATION}

A study was conducted to assess quantitative changes in physical characteristics and chemical constituents during ripening of 'Granny Val' grapes picked at three stages of maturity: green, table, and ripe. The studied variables were: soluble solids (SS), titratable acidity (AT), SS/AT, sugars (glucose, sucrose and fructose), phenols, color and firmness. Most of the physical and chemical parameters evaluated increased: soluble solids SS (9.50 to12.80), Ratio SS/TA (2.06 to 5.17), pH (3.14 to 3.44) and phenolic compounds (25.23 to 31.96 ) while titratable acidity (AT) declined by $47 \%$ (4.64 to 2.48). Compression force (firmness) decreased by $48 \%$ and penetration force also did by $14 \%$, Hunter $\mathrm{L}^{*}$ (brightness), and $\mathrm{b}^{*}$ decreased, while Hunter $\mathrm{a}^{*}$ increased. The grapes became less green (more yellow) and bronze in color. Firmness of the fruit was reduced in $48 \%$ and of the peel in $14 \%$ while ripening. However, they kept good qualities with the ripeness process which allow them to resist during storage and transportation.

KEY-WORDS: GRAPES; Vitis rotundifolia; RIPENING; FRUIT QUALITY.

\section{REFERENCIAS}

1 ANDERSEN, P. C. Performance of 20 muscadine grape cultivars in North Florida. Journal of the American Pomological Society, v. 60, n. 3, p. 129-135, 2006.

2 ASENJO, C. Acerola. In: SHAW, E. (ed.) Tropical and subtropical fruits. Wesport, Conneticut: AVI Publishing, 1980. p. 341-374.

3 BAUSCH \& LOMB S/F. ABBE-3L: refractometer instructions. $2^{\text {nd }}$ ed. Rochesterm, New York, 2000. 22 p.

4 BASHA, S. M.; VASANTHAIAH, H.K.N.; KAMBIRANDA, D.M.; EASWARAN, K.Q. G. Genetic variation in sugar composition among muscadine, Florida hybrid bunch and bunch grape genotypes. International Journal of Wine Research, v. 4, p.15-23, 2012. 
5 BRADY, P.L.; THOMSEN, M.; MORRIS, J.R. Marketing options for grapes and grapes products. Fayetteville: Arkansas Agricultural Experiment Station/Division of Agriculture/University of Arkansas, 2010. (Res. Report, 988).

6 BREMAN, J. W.; SIMONNE, A.; HOCHMUTH, R.C.; LANDRUM, L.; TAYLOR, M.; EVANS, K.; PEAVY, C.; GOODE, D. Quality characteristics of selected muscadine grape cultivars grown in North Florida. Proc. Fla. State Hort. Soc., v. 120, p. 8-10, 2007.

7 BUBOLA, M.; PERŠURIĆ, D. Yield components, vegetative growth and fruit composition of 'Istrian Malvasia' (Vitis vinifera L.) as affected by the timing of partial defoliation. Agriculturae Conspectus Scientificus, v. 77, n. 1, p. 21-26, 2012.

8 CALlejas ROdRígueZ, R.; PEPPI ARONOWSKY, C.; BRAYOVIC PIÑONES, M.; KANIA KUHL, E. Categorías de firmeza de bayas en diferentes variedades de uva de mesa (Vitis vinifera L.). Revista de la Facultad de Ciencias Agrarias, v. 43, n. 1, p. 127-141. 2011.

9 CARROL, D.E.; HOOVER, M.W.; NESBITT, W.V. Sugar and organic acid concentrations in cultivars of muscadines grapes. J. Am. Soc. Hort., v. 96, p. 737-738, 1971.

10 CHAMUL, R. S. Process mass and energy balance, physico-chemical changes during process and storage, and pigment composition of bronze muscadine grape juice. 1990. 160 p. Thesis (Food Science and Technology), Mississippi State University, Starkville, 1990.

11 CHAOVANALIKIT, A.; WROLSTAD, R. Anthocyanin and polyphenolic composition of fresh and processed cherries. J. Food. Sci., v. 69, n. 1, p. 73-83, 2004a.

12 CHAOVANALIKIT, A.; Y WROLSTAD, R. Total anthocyanins and total phenolics of fresh and processed cherries and their antioxidant properties. J. Food Sci., v. 69, n. 1, p. 67-72, 2004b.

13 CHIAROTTI, F.; GUERIOS, I.T.; CUQUEL, F.L.; BIASI, L.A. Melhoria da qualidade de uva 'Bordô' para produção de vinho e suco de uva. Rev. Bras. Frutic., v. Especial E, p. 618-624, 2011.

14 CONNER, P.J. Performance of muscadine grape cultivars in Southern Georgia. Journal of the American Pomological Society, v. 63, n. 3, p. 101-107, 2009.

15 DOREYAPPA, G.; KUDDAR, A.G. Studies on ripening in mango (Mangifera indica L.). Fruit. J. Food Sci., v. 38, n. 2, p. 135-137, 2001.

16 FILS-LYCAON, B.; BURET, M. Loss of firmness and changes in pectic fractions during ripening and overripening of sweet cherry. Hort. Science, v 25, n. 7, p. 777-778, 1990.

17 FISHER SCIENTIFIC. Instructions Fisher Accumet Model 925 Meter. Pittsburg, Pennsylvania, 1987. (Bulletin 7-1019-n).

18 FLORA, L.F.; LANE, R.P. Effects of ripeness and harvest date on several physical and compositional factors of Cowart muscadine grapes. Am. J. Enol. Vitic., v. 30, p. 241-246, 1979.

19 FRANKEL, E.N.; WATERHOUSE, A.L.; TEISSEDRE, P.L. Principal phenolic phytochemicals in selected Californian wines and their antioxidant activity in inhibiting oxidation of human low density lipoproteins. J. Agric. Food Chem., v. 43, p. 890894, 1995.

20 GONZÁLEZ-SAN JOSÉ, M.L.; BARRON, L.J.; JUNQUERA, B.; ROBREDO, M. Application of principal components analysis to ripening indexes for wine grapes. J. Food Comp. Anal., v. 4, p. 245-255, 1991.

21 HRAZDINA, G.; PARSONS, G. F.; MATTICK, L.R. Physiological and biochemical events during development and maturation of grape berries. Am. J. Enol. Vitic., v. 35, p. 220-227, 1984.

22 HUANG, J. X. Postharvest handling of muscadine grapes (Vitis rotundifolia Michx.) and rabiteye blueberry (Vaccinium ashei Reade). 1996. 270 p. Thesis (Plant and Soil Sciences), Mississippi State University, Starkville, 1996.

23 INFANTE, R. Polifenoles del vino y oxidabilidad de las lipoproteínas ¿blanco o tinto? Clin. Invest. Arterioesclerosis, v. 9, p. 19-22, 1997.

24 INSTRON CORP. Texture evaluation of apples. Canton, Massachusetts, 1972.

25 JEANDET, P.; BESSIS, R.; SBAGHI, M.; MEUNIER, P.; TROLLAT, P. Resveratrol content in wines of different ages: relationship with fungal disease pressure in the vineyard. Am. J. Enol. Vitic., v. 46, p.1-4, 1995.

26 JOGAIAH, D.; OULKAR, D.P.; VIJAPURE, A.N.; MASKE, S.R.; SHARMA, A.K.; SOMKUWAR, R.G. Influence of canopy management practices on fruit composition of wine grape cultivars grown in semi-arid tropical region of India. Afr. J. Agric. Res., v. 8, n. 26, p. 3462-3472, 2013.

27 KALT, W. Effects of production and processing factors on mayor fruits and vegetables antioxidants. J. Food Science, $v$. 70, n. 1, p. 11-19, 2005. 
28 KENNEDY, J. Understanding grape berry development. Practical Winery \& Vineyard Journal July/August. Disponible en: http//www.practicalvineyard.com/JulyAugust02/julaug02p14.html. Visitado el: 12 de enero de 2005.

29 KINSELLA, J.; FRANKEL, E.; GERMAN, B.; KANNER, J. Possible mechanism for protective role of antioxidants in wine and plant foods. Food Technol., v. 47, n. 4, p. 85-89, 1993.

30 KODUR, S. Effects of juice $\mathrm{pH}$ and potassium on juice and wine quality, and regulation of potassium in grapevines through rootstocks (Vitis): a short review. Vitis, v. 50, n. 1, p. 1-6, 2011.

31 LAGUADO, N.; MARIN, M.; ARENAS, M. L. de; CASTRO, R.C. de. Relación entre variables indicadoras de maduración de frutos de guayabo (Psidium guajava L.) var. Dominicana Roja. Rev. Fac. Agron. (LUZ), v. 15, p. 422-428, 1998.

32 LANIER, M. R.; MORRIS, J. R. Evaluation of density separation for defining fruit maturities and maturation rates of onceover harvested muscadine grapes. J. Am. Soc. Hort. Sci., v. 104, p. 249-252, 1979

33 LIANG, M.; CHEN, L.H.; HEGWOOD, C.P.JR. Physical and mechanical properties of Muscadines grapes. Starkville: Divison of Agriculture, Forestry, and Veterinary Medicine, Mississippi State University, 1991. (Technical Bulletin, 176).

34 MAHDAVI, M.; NIKNIAZ, Z.; RAFRAF, M.; JOUYBAN, A. Determination and comparison of total polyphenol and vitamin C contents of natural fresh and commercial fruit juices. Pakistan Journal of Nutrition, v. 9, n. 10, p. 968-972, 2010.

35 MASCARENHAS, R. de J.; SILVA, S. de M.; LOPES, J.D.; COÊLHO DE LIMA, M. A. Avaliação sensorial de uvas de mesa producidas no vale do São Francisco e comercializadas em João Pessoa - PB. Rev. Bras. Frutic., v. 32, n. 4, p. 993-1000, 2010

36 MASCARENHAS, R. de J.; SILVA, S. de M.; COÊLHO DE LIMA, M.A.; NUNES MENDONÇA, R.J.; HOLSCHUH, H.J. Characterization of maturity and quality of Brazilian apirenic grapes in the São Francisco river Valley. Ciênc. Tecnol. Aliment., v. 32, n. 1, p. 26-33, 2012.

37 MORRIS, J. Handling and marketing of muscadines grapes. Fruit South, v. 4, n. 2, p. 12-14, 1980.

38 MORRIS, J.R.; BRADY, P.L. The Muscadine experience: adding value to enhance profits. Fayetteville: Arkansas Agricultural Experiment Station/Division of Agriculture/University of Arkansas System, 2004. p. 76. (Research Report, 974).

39 OJEDA, M.; PIRE, R. Estrategias para estimar el nivel de maduración en uvas para vinificación. Bioagro, v. 9, n. 1, p. $20-25,1997$

40 PHILILP, T.; KUYENDA, J.R. Changes in titratable acidity, ${ }^{\circ}$ Brix, pH potassium content, malate and tartrate during berry development of 'Thompson Seedless' grapes. J. Food Sci., v. 38, p. 874-876, 1973.

41 POMERANZ, Y.; MELOAN, C.E. Food analysis: theory and practice. Westport, CT: AVI Publishing, 1978.

42 REDGWELL, R.J.; FISCHER, M.; KENDALL, E.; MAC-RAE, E.A. Galactose loss and fruit ripening: high - molecular mass arabinogalactans in the pectic polysaccharides of the fruit cell walls. Planta, v. 203, p. 174-181, 1997.

43 ROMERO-PEREZ, A.I.; LAMUELA-REVENTS, R.M.; ANDRES-LACUEVA, C.; DE LA TORRE-BORONAT, M.C. Method for the quantitative extraction of resveratrol and piceid isomers in grape berry skins. Effect of powdery mildew on the stilbene content. J. Agri. Food Chem., v. 49, n. 1, p. 210-215, 2001.

44 SAS Institute Inc. SAS user's guide basics. Version 5. Cary, NC, 1997.

45 SIEMANN, E.H.; CREASY, L.L. Concentration of phytoalexin resveratrol in wine. Am. J. Enol. Vitic., v. 43, p. 49-52, 1992.

46 SIMONNE, E.; SARGENT, S.A.; STUDSTILL, D.; SIMONNE, A.; HOCHMUTH, R.; KERR, S. Field performance, chemical composition and sensory evaluation of grape tomato varieties. Proc. Fla. Hort. Soc., v. 119, p. 376-378, 2005.

47 SIMONETTI, P.; PIETTA, P.; TETOLIN, G. Polyphenol content and total antioxidant potential of selected Italian wines J. Agric. Food Chem., v.45, p.1152-1155, 1997.

48 SINGLETON, V.L.; ROSSI, J.A. Colorimetry of total phenolic with phosphomolybdic-phosphotungstic acid reagents. Am. J. Enol. Vitic., v. 16, n. 3, p. 144-158, 1965.

49 Statistical Package for Social Sciences (SPSS). Texture evaluation of apples. Canton, MA: Instron Corp., 1972.

50 STEEL, R.G.D.; TORRIE, J.M. Principles and procedures of statistics. New York: McGraw-Hill Book, 1985

51 STRIEGLER, R.K.; MORRIS, J.R.; CARTER, P.M.; THREFALL, R.T.; HOWARD, L. R. Yield, quality, and nutraceutical potential of selected muscadine cultivars grown in southwestern Arkansas. HortTechnology, v. 15, p. 276-284, 2005.

52 STRINGER, D.J.; MARSHALL, D.A.; SAMPSON, B.J.; SPIERS, J.M. Performance of muscadine grape cultivars in 
Southern Mississippi. HortTechnology, v. 18, p. 726-733, 2008.

53 WALKER, T.R.; MORRIS, J.R.; THRELFALL, R.T.; MAIN, G.L.; LAMIKANRA, O.; LEONG, S. Density separation, storage, shelf life, and sensory evaluation of 'Fry' muscadine grapes. Hortscience, v. 36, n. 5, p. 941-945, 2001.

54 WATSON, B. Evaluation of vinegrape maturity. In: HELLMAN, E.W. (Ed). Oregon viticulture. Corvallis: Oregon State University Press, 2003. p. 235-245.

55 WOODS, A.E.; AURAND, L.W. Laboratory manual in food chemistry. Westport, CT: AVI Publishing, 1977.

56 ZOECKLEIN, B. W. Grape sampling and maturity evaluation for growers. Vintner's Corner v. 16, n. 1. Disponible en: http://www.apps.fst.vt.edu/extension/enology/VC/ Jan-Feb01.html. 2001. Visitado en: 13 de mayo de 2005.

\section{AGRADECIMIENTOS}

Al Dr. James Garner Jr. Profesor Emeritus de Plant and Soil Sciences, por su asesoría y colaboración en el Laboratorio de "Horticultural Sciences". MSU, MS. USA. 